\title{
Research Progress on Functional Activity and Preparation of Low
}

\section{Molecular Weight Polysaccharides}

\section{Xue-Jun LIU ${ }^{1, a}$, Yu XU ${ }^{1, b}$, Chang ZHU ${ }^{2, c}$, Jia-Hui WANG ${ }^{1, d}$, Xuan ZHANG ${ }^{1, e}$, Rui TANG ${ }^{1, f}$, Xue-Jun LIU*}

${ }^{1}$ College of Food Science and Engineering, Jilin Agricultural University, Changchun, Jilin, 130118, China

${ }^{2}$ Jilin Engineering Vocational College, Food Engineering Branch, Siping, Jilin,136000, China

aliuxuejun63@163.com, b1422491537@qq.com, '14170567@qq.com, d412491945@qq.com,

e287863920@qq.com, ${ }^{f} 411649317 @ q q . c o m, ~ * l i u x u e j u n 63 @ 163 . c o m$

Key words: Low molecular weight polysaccharides; Preparation methods; Functional activities; Research progress

Abstract. This review introduced the low molecular weight polysaccharides preparation methods, and the research progress of functional activity of low molecular weight polysaccharides were described, and the future development and application prospect of the low molecular weight polysaccharides were discussed, and we put forward to the research of low molecular weight polysaccharides should focus on functional food and clinical application , and contribute our own strength for clinical medicine.

\section{Introduction}

The studies showed that polysaccharides were a class of important bioactive substances, which had a variety of activities ${ }^{[1]}$, and had wide applications in functional food and medicine. Although there are a lot of biological activity of large molecular weight polysaccharides, but because of its large molecular weight, poor solubility, apparent viscosity and not easy to be absorbed, so they were very limited in the application ${ }^{[2]}$. The results showed that the low molecular weight polysaccharides had good solubility, low viscosity and easy absorption. Therefore, the research of low molecular weight polysaccharides were in line with the needs of modern society for polysaccharides, which is of great significance for the effective utilization of polysaccharides and the improvement of polysaccharides utilization value.

\section{Preparation of Low Molecular Weight Polysaccharides}

\section{(1) Degradation method}

At present, the main methods of polysaccharides degradation are chemical degradation, physical degradation and biodegradation ${ }^{[3]}$. Zhang ${ }^{[4]}$ et al. used free radical degradation on tremella polysaccharides, and their antioxidant activity were explored. The results showed that the antioxidant activity of low molecular weight tremella polysaccharides were higher. The results indicated that the degradation of free radical was an effective method to decrease the molecular weight of polysaccharides. $\mathrm{Li}^{[5]}$ et al. used microwave assisted acid hydrolysis to degrade polysaccharides from Porphyra yezoensis and greatly increased the degradation rate. Zhou ${ }^{[6]}$ et al. also used ultrasonic methods to treat the polysaccharides from Porphyra yezoensis. The results 
showed that ultrasonic degradation greatly enhanced the biological activity, which indicated that the ultrasonic wave was beneficial to the degradation of polysaccharides.

\section{(2)Column chromatography}

At present, some people have obtained low molecular weight polysaccharides by column chromatography. $\mathrm{Liu}^{[7]}$ et al. used macroporous resin column chromatography on the crude polysaccharides of Ganoderma lucidum, the elution was then concentrated and subjected to column chromatography, and the major eluted fraction was further purified by gel-filtration chromatography. Two single peaks were collected, two low molecular weight Ganoderma lucidum polysaccharides were obtained with average molecular weights. $\mathrm{Cui}^{[8]}$ et al. extracted polysaccharides by using $95 \%$ alcohol and used DEAE-SepharoseCL-6B gel chromatography, separated and purified a novel water-soluble polysaccharides. And the low molecular weight polysaccharides were obtained by high performance liquid chromatography (HPSEC) and the molecular weight was 50KDa.

\section{(3) Hot water extraction}

$\mathrm{Liu}^{[9]}$ et al. researched extraction and antioxidant activity study of low molecular weight gracilaria polysaccharides. She used orthogonal experiments to study hot water extraction and ethanol precipitation of gracilaria low molecular weight polysaccharides. Zhang ${ }^{[10]}$ et al. used hot water extraction in the primary study of extraction, purification and structure of low molecular weight pumpkin polysaccharides.

\section{Functional activity of low molecular weight polysaccharides}

\section{(1) Antioxidant activity of low molecular weight polysaccharides}

It has been reported that low molecular weight polysaccharides have strong antioxidant activity. Zhang $^{[1]}$ et al. prepared three low molecular weight samples from shrimp and studied their antioxidant activity. The results showed that LP3 had higher scavenging effect on hydroxyl and superoxide radicals than the other two degradation samples, and the molecular weight was lower and the antioxidant activity was stronger. Sheng ${ }^{[12]}$ et al. successfully obtained four low molecular polysaccharides and studied their antioxidant activity in vitro. The results showed that all polysaccharides indicated significant antioxidant activity. Therefore, low molecular weight polysaccharides seemed to play an important role in the exploration of natural antioxidants in the food industry and medicine.

\section{(2)Antitumor activity of low molecular weight polysaccharides}

The researchers also confirmed the antitumor activity of low molecular weight polysaccharides. Sun $^{[13]}$ et al. isolated extracellular polysaccharides (EPSs) from Porphyridium algae and then degraded by microwave and $\mathrm{H}_{2} \mathrm{O}_{2}$ under ultrasonic waves, six products were obtained. Antitumor activity of different molecular weight polysaccharides was assessed by in vivo S180-tumor bearing mouse model. Different doses of EPS inhibited the growth of implanted S180 tumors. Seoa ${ }^{[14]}$ et al. prepared low molecular weight polysaccharide in ginseng, which activated macrophage RAW264.7, induced apoptotic cell death of melanoma cells and inhibited the growth of skin melanoma cells.

\section{(3)Hypoglycemic activity of low molecular weight polysaccharides}

In fact, low molecular weight polysaccharides had good hypoglycemic activity. Chen $^{[15]}$ et al. used hyperglycemia mouse model to verify the hypoglycemic activity of low molecular weight Cordyceps polysaccharides in vivo. The results showed that the low molecular weight Cordyceps sinensis polysaccharides showed better hypoglycemic effect, and they could significantly reduce the blood glucose of diabetic mice and could improve the mouse glucose tolerance abnormalities within a certain range, at the same time, low molecular weight Cordyceps sinensis polysaccharides could 
effectively reduce the high blood sugar on the kidney and liver damage and relieve the weight loss caused by glucose metabolism disorders.

\section{(4) Immunomodulatory activity of low molecular weight polysaccharides}

Low molecular weight polysaccharides also had a strong immune regulatory activity. Dai ${ }^{[16]}$ isolated and purified to obtain a new low molecular weight astragalus polysaccharides(LMw-APS) from astragalus, and their immunological activity was explored. The results showed that LMw-APS could significantly promote the proliferation of splenic lymphocytes induced by ConA and LPS, and could significantly promote the production of antibody in mouse antiserum. The results provided a theoretical basis for the development of LMw-APS as a special type of immune adjuvant.

\section{(5) Renal protective effect of low molecular weight polysaccharides}

In addition, low molecular weight polysaccharide had a protective effect on the kidney. $\mathrm{Li}^{[17]}$ et al. extracted fractions of low molecular weight sulfated polysaccharides(LMWSP) from Laminaria japonica and studied renal protective effects of glycerol on acute kidney injury (AKI) induced by glycerol in rats. The results showed that glycerol treatment significantly increased serum creatinine (SCr) and blood urea nitrogen (BUN) levels. Intraperitoneal injection of LMWSP fractions significantly reduced SCr and BUN levels and reduced renal swelling.

\section{Expectation}

At present, the research on low molecular weight polysaccharide had the following three shortcomings: First of all, most people focused on the extraction, purification, activity of low molecular weight polysaccharides, compared with the high molecular weight polysaccharides, the mechanism of low molecular weight polysaccharides with higher activity was unclear, researchers in the future should focus on the research of the micro mechanism of their activity; Secondly, the study of low molecular weight polysaccharides were now limited to the laboratory stage, they should be gradually applied to functional foods and clinical practice to make them really play a role and improve utilization value; In addition, it is the direction of the scientific research workers how to make the industrialization and produce economic benefits.

\section{Conclusions}

This review summarizes the research progress on the preparation methods and functional activities of low molecular weight polysaccharides, and the future development direction of low molecular weight polysaccharides were also researched. Study of low molecular weight polysaccharides could improve the biological activity and the application range of polysaccharides, which had great research prospect. It is of great significance to improve the utilization value of polysaccharides in the development and clinical application of new drugs and health foods.

\section{References}

[1] L Shi. Bioactivities, isolation and purification methods of polysaccharides from natural products: A review[J]. International Journal of Biological Macromolecules.92:37-48(2016)

[2] D Shi, W Liu, Y Liu, R Xu, L Tang, Z Li, T Lu. Antitumor activity and preparation of low molecular weight fucoidan polysaccharides[J]. Shizhen Medical Journal of Medicine. 23(1):53-55 (2012).In Chinese 
[3] L Chen, H Wu. Research progress of polysaccharide degradation method [J]. Journal of Chinese traditional medicine. 26(1):133-135 (2008). In Chinese

[4] Z Zhang, X Wang, M Zhao, H Qi. Free-radical degradation by $\mathrm{Fe}^{2+} / \mathrm{Vc} / \mathrm{H}_{2} \mathrm{O}_{2}$ and antioxidant activity of polysaccharide from Tremella fuciformis[J]. Carbohydrate Polymers. 112:578-582 (2014)

[5] B Li, S Liu, R Xing, K Li, R Li, Y Qin, X Wang, Z Wei, P Li. Degradation of sulfated polysaccharides from Enteromorpha prolifera and their antioxidant activities[J]. Carbohydrate Polymers. 92(2):1991-1996 (2013)

[6] C Zhou, X Yu, Y Zhang, R He, H Ma. Ultrasonic degradation, purification and analysis of structure and antioxidant activity of polysaccharide from Porphyra yezoensis Udea[J]. Carbohydrate Polymers. 87(3):2046-2051 (2012). In Chinese

[7] W Liu, H Wang, X Pang, W Yao, X Gao. Characterization and antioxidant activity of two low-molecular-weight polysaccharides purified from the fruiting bodies of Ganoderma lucidum[J]. International Journal of Biological Macromolecules. 46(4):451-457 (2010)

[8] L Cui, G Wang, L Sun, Y Liu, Y Xiao, H Xu, J Liu. Study on Immune Activity of Low Molecular Weight polysaccharides of Agaricus [J]. Journal of Traditional Chinese Medicine. 30(2):10-13 (2013). In Chinese

[9] H.Y. Liu, M Zhao. Studies on Extraction and Antioxidant Activity of Low Molecular Weight Polysaccharides from Gracilaria sylvestris[J]. Food Science and Technology. 26(3):67-69 (2005). In Chinese

[10] F Zhang, B Shi, S Zhang. Preparation, Purification and Structure of Low Molecular Weight Pumpkin Polysaccharides[J]. Food Science and Technology. 29(3):93-95 (2008). In Chinese

[11] Z Zhang, X Wang, C Liu, J Li. The degradation, antioxidant and antimutagenic activity of the mucilage polysaccharide from Dioscorea opposita[J]. Carbohydrate Polymers. 150:227-231 (2016)

[12] J Sheng, Y Sun. Antioxidant properties of different molecular weight polysaccharides from Athyrium multidentatum (Doll.) Ching[J]. Carbohydrate Polymers. 108:41-45 (2014)

[13] L Sun, L Wang, Y Zhou. Immunomodulation and antitumor activities of different-molecular-weight polysaccharides from Porphyridium cruentum[J]. Carbohydrate Polymers. 87(2):1206-1210 (2012)

[14] J.Y. Seoa, C.W. Leea, D.J. Choia, J Leea, J.Y. Leeb, Y Park. Ginseng marc-derived low-molecular weight oligosaccharide inhibits the growth of skin melanoma cells via activation of RAW264.7 cells[J]. International Immunopharmacology. 29(2):344-353 (2015)

[15] C Chen, Z Zhu, L Chen. Study on hypoglycemic activity of polysaccharides with low molecular weight Cordyceps militaris[J]. Modern Food Science and Technology. 33(4):1-7 (2017). In Chinese

[16] L Dai. Separation and Purification of Low Molecular Weight Astragalus Polysaccharides and Its Immunological Activity [D]. Northeast Normal University. In Chinese

[17] X Li, J Wang, H Zhang, Q Zhang. Renoprotective effect of low-molecular-weight sulfated polysaccharide from the seaweed Laminaria japonica on glycerol-induced acute kidney injury in rats[J].International Journal of Biological Macromolecules. 95:132-137 (2017) 